\title{
Regulation of Neutrophil Apoptosis Differs after in vivo Transmigration to Skin Chambers and Synovial Fluid: A Role for Inflammasome-Dependent Interleukin-1 $\beta$ Release
}

\author{
Karin Christenson Lena Björkman Anna Karlsson Johan Bylund \\ The Phagocyte Research Laboratory, Department of Rheumatology and Inflammation Research, Sahlgrenska \\ Academy at University of Gothenburg, Gothenburg, Sweden
}

\section{Key Words}

Phagocyte · Granulocyte · Apoptosis · Transmigration · Skin

chamber $\cdot$ Exudate $\cdot$ Rheumatoid arthritis $\cdot$ Synovia feature of tissue neutrophils and imply that autocrine IL-1 $\beta$ signaling could be an important factor in determining how life and death of neutrophils is regulated in inflamed tissues.

Copyright $\odot 2013$ S. Karger AG, Basel

\section{Introduction}

Transmigration of neutrophils from the bloodstream to an inflamed tissue is a complex process whereby the cells typically change from a resting state in circulation into a preactivated, or primed, state when reaching the inflamed site [1]. The primed state is characterized by a hyperresponsiveness to stimulation brought about in part by exocytosis of intracellular granules that results in upregulation of surface receptors $[2,3]$. In addition, tissue neutrophils are often primed with regard to de novo synthesis of cytokines; most notably, the genes for proinflammatory cytokines interleukin (IL)- 8 and IL- $1 \beta$ are highly upregulated in skin chamber neutrophils [4]. High levels of these and other cytokines are also found in skin chamber fluid [5-7].

Neutrophils are potent microbicidal cells packed with toxic substances aimed at microbial killing. However, these toxic substances may also inflict endogenous tissue damage and it is therefore crucial that the activity and

\section{KARGER}

E-Mail karger@karger.com

www.karger.com/jin
Dr. Karin Christenson

Department of Rheumatology and Inflammation Research, Sahlgrenska Academy at University of Gothenburg, Guldhedsgatan 10 SE-413 46 Gothenburg (Sweden)

E-Mail karin.christenson@ rheuma.gu.se 
longevity of these cells is tightly controlled; the latter is in part ascertained by a high propensity to undergo spontaneous apoptosis. Apoptotic cells are nonfunctional, but structurally intact, i.e. they retain their tissue-damaging/ proinflammatory substances intracellularly and by this minimize the risk of collateral damage to surrounding tissues. In addition, dead cells are rapidly cleared by other phagocytes, primarily macrophages [8], a process typically associated with a pronounced release of anti-inflammatory cytokines from the engulfing macrophages [9]. Thus, in order to regain homeostasis after acute inflammation, it is crucial that both apoptosis and clearance are smoothly executed; disturbances in these events can contribute to prolonged or chronic inflammation [10]. Concerning human neutrophils, apoptosis has almost exclusively been studied in cells isolated from peripheral blood. These cells can be modulated in both directions regarding death rate; whereas proapoptotic factors accelerate cell death, a wide variety of antiapoptotic factors extend viability [11]. We recently showed that in vivo transmigrated human neutrophils, isolated using experimental skin chamber methodology on healthy subjects, were completely resistant to antiapoptotic stimulation that potently expand the life span of cells purified from peripheral blood [5].

In this study, we set out to investigate whether the priming status of the skin chamber neutrophils has bearing on the regulation of apoptosis, but also to assess how neutrophils regulate cell death after in vivo transmigration to a pathologically inflamed tissue. We found that in stark contrast to cells isolated from skin chambers, in vivo transmigrated neutrophils from synovial fluid of patients with inflammatory arthritis were perfectly able to respond to antiapoptotic stimulation when cultured in vitro. In a coculture set-up, we found that skin chamber neutrophils prolonged the life span of blood neutrophils and identified IL- $1 \beta$, continuously secreted by skin chamber neutrophils, as a soluble factor potentially responsible for this effect. Downregulation of inflammasome-mediated IL- $1 \beta$ secretion through inhibition of caspase 1 decreased the death-delaying signal released during coculture and also enhanced apoptosis of skin chamber neutrophils. This implies that skin chamber neutrophils are under constant death suppression due to autocrine IL-1 $\beta$ signaling. Our results suggest that matters of neutrophil life and death are regulated differently depending on the site and inflammatory setting into which the cells transmigrate and indicate a role for inflammasome-mediated IL- $1 \beta$ in dictating these processes.

\section{Materials and Methods}

\section{Reagents}

Dextran was purchased from Pharmacosmos (Holbaek, Denmark) and Ficoll-Paque from Fischer Scientific GTF AB (Gothenburg, Sweden). Recombinant human serum amyloid A (human Apo-SAA; rSAA) was from PeproTech Inc. (Rocky Hill, N.J., USA). The caspase 1 inhibitor Ac-YVAD-CMK (YVAD) was from Cayman Chemical (Ann Arbor, Mich., USA). Purified peptidoglycan (PGN) and lipoteichoic acid (LTA), both from Staphylococcus aureus, the synthetic triacylated lipopeptide Pam3CSK $4\left(\mathrm{P}_{3} \mathrm{C}\right)$ and IL- $1 \beta$ were all from Invivogen (San Diego, Calif., USA). The IL-1 receptor antagonist (IL-1RA) and IL-8 were from R\&D Systems (Minneapolis, Minn., USA). Lipopolysaccharide (LPS) from Escherichia coli (serotype O111:B4) was from Sigma-Aldrich (St. Louis, Mo., USA) as was recombinant human granulocyte macrophagecolony stimulating factor (GM-CSF). Functional grade, monoclonal anti-CD95 antibody (FASL) was from Nordic BioSite (Täby, Sweden). Annexin V-FLUOS was from Roche Diagnostics (Mannheim, Germany) and 7-amino-actinomycin D (7-AAD) from BD Bioscience (Stockholm, Sweden).

\section{Preparation of Cells}

After informed consent, peripheral blood and synovial fluid were drawn from patients with inflammatory arthritis (table 1). Within $2 \mathrm{~h}$ from the time of aspiration, synovial fluid was filtrated through 40- $\mu \mathrm{m}$ nylon cell strainers (BD Biosciences, Bedford, Mass., USA) before centrifugation at $335 \mathrm{~g}$ for $10 \mathrm{~min}$. The cell pellet was resuspended in Krebs-Ringers phosphate buffer with glucose supplemented with $\mathrm{Ca}^{2+}(1 \mathrm{mM})$ and stored on melting ice. All cell preparations were directly analyzed by flow cytometry and the relative abundance of neutrophils was determined based on size and granularity after gating away cellular debris. Only patients with a neutrophil content of at least $65 \%$ in their synovial fluid were included in the study (table 1).

Blood neutrophils were separated by dextran sedimentation and Ficoll-Paque centrifugation according to Boyum et al. [12, 13]. After separation, the neutrophils were washed twice, resuspended in Krebs-Ringers phosphate buffer with glucose supplemented with $\mathrm{Ca}^{2+}(1 \mathrm{mM})$ and stored on melting ice until use.

Skin chamber neutrophils were obtained as described [5] from healthy volunteers who also donated peripheral blood (from which blood neutrophils were prepared as stated above). The study was approved by the regional ethical board (No. S010-03 and 543-07).

Basic Neutrophil Culture and Evaluation of Cell Death

Neutrophils $\left(5 \times 10^{6} / \mathrm{ml}\right)$ were cultured in RPMI-1640 supplemented with $10 \%$ FCS and 1\% penicillin/streptomycin for $30 \mathrm{~min}$ before addition of medium, LPS (100 ng/ml), rSAA $(2 \mu \mathrm{M})$, GMCSF (100 ng/ml), PGN (10 $\mu \mathrm{g} / \mathrm{ml})$, LTA $(10 \mu \mathrm{g} / \mathrm{ml}), \mathrm{P}_{3} \mathrm{C}(100 \mathrm{ng} /$ $\mathrm{ml})$ or FASL $(10 \mu \mathrm{g} / \mathrm{ml})$ and thereafter further cultured for the indicated time $\left(37^{\circ} \mathrm{C}\right.$ with $\left.5 \% \mathrm{CO}_{2}\right)$.

Quantification of caspase 3/7 activity was performed on freshly prepared cells $\left(10^{5}\right.$ cells per sample) or on cells cultured for 3.5 $\mathrm{h}$ with addition of medium or FASL $(10 \mu \mathrm{g} / \mathrm{ml})$ using a CaspaseGlo 3/7 kit (Promega Corporation, Madison, Wisc., USA) and analyzed on a LB940 Mithras multilabel reader (Berthold Technologies, Bad Wildbad, Germany).

Evaluation of cell death was performed essentially as described in Christenson et al. [11]. In short, cells were stained with annexin 
Table 1. Description of patients contributing synovial fluid and peripheral blood to the study

\begin{tabular}{|c|c|c|c|c|c|c|c|}
\hline $\begin{array}{l}\text { Patient } \\
\text { No. }\end{array}$ & $\begin{array}{l}\text { PMN in synovial } \\
\text { fluid, \% }\end{array}$ & Diagnosis & Treatment & $\begin{array}{l}\text { Duration of in- } \\
\text { flammatory epi- } \\
\text { sode }^{1}\end{array}$ & $\begin{array}{l}\text { ESR } \\
\mathrm{mm}^{2}\end{array}$ & $\begin{array}{l}\text { Age } \\
\text { years }\end{array}$ & Gender \\
\hline 1 & 91 & RA & azathioprine, corticosteroid & 1 week & 110 & 36 & female \\
\hline 2 & 69 & RA & methotrexate & 10 days & - & 70 & female \\
\hline 3 & 74 & $\mathrm{OlA}$ & azathioprine, corticosteroid & $1-2$ weeks & 115 & 34 & female \\
\hline 4 & 83 & MA & methotrexate & 2 weeks & 52 & 44 & male \\
\hline 5 & 92 & SpA & sulfasalazine, corticosteroid & $1-2$ weeks & 100 & 49 & male \\
\hline 6 & 74 & SpA & methotrexate & 2 weeks & 59 & 79 & female \\
\hline 7 & 73 & RA & methotrexate, infliximab & 2 weeks & - & 24 & female \\
\hline 8 & 80 & RA & etanercept, leflunomide & 2 weeks & 85 & 55 & female \\
\hline 9 & 66 & SpA & methotrexate, infliximab & 2 weeks & - & 34 & male \\
\hline 10 & 92 & RA & sulfasalazine & 3 weeks & - & 55 & female \\
\hline 11 & 75 & SpA & sulfasalazine & 2 weeks & - & 35 & male \\
\hline 12 & 73 & RA & - & 1 week & 40 & 64 & female \\
\hline 13 & 90 & $\mathrm{OlA}$ & corticosteroid & 2 weeks & 2 & 53 & male \\
\hline
\end{tabular}

PMN = Polymorphonuclear neutrophils; ESR = erythrocyte sedimentation rate; RA = rheumatoid arthritis; $\mathrm{OlA}=$ oligoarthritis; $\mathrm{MA}=$ monoarthritis; $\mathrm{SpA}=$ spondyloarthritis.

${ }^{1}$ Self-evaluation of the time from onset of synovitis to aspiration of synovial fluid.

${ }^{2}$ Erythrocyte sedimentation rate (normal levels, men $<15 \mathrm{~mm}$, women $<20 \mathrm{~mm}$ ) at time of sampling.

$\mathrm{V}$ in combination with 7-AAD and analyzed by flow cytometry on a FacsScan or Accuri C6 (Becton Dickinson, Mountain View, Calif., USA). All data were analyzed using WinMDI 2.9 or CFlow plus software. Levels of necrosis (annexin V/7-AAD double-positive events) were overall very low, and therefore, the term 'apoptotic', if not stated differently, refers to all annexin-V-positive cells.

\section{Coculture Experiments}

Freshly isolated neutrophils from peripheral blood were directly labeled with a VybrantV CFDA SE cell tracker kit (Invitrogen, Carlsbad, Calif., USA), according to the manufacturer's instructions, using a dye concentration of $0.5 \mu \mathrm{M}$. The labeled cells were then cocultured with unlabeled skin chamber neutrophils from the same subject. After $20 \mathrm{~h}$ of coculture, apoptosis was monitored by annexin-V-APC staining and analysis by flow cytometry after gating on carboxyfluorescein diacetate (CFDA)-positive neutrophils. In parallel experiments, labeled and unlabeled cells were cultured separately. The cell tracker dye per se did not affect neutrophil viability or the ability of cells to respond to antiapoptotic factors (data not shown). The proportion of blood versus skin chamber neutrophils remained constant over the time of coculture (data not shown), indicating that no asymmetrical loss of cells (due to e.g. cellular disintegration) had occurred; we also did not see, by confocal microscopy, any signs of neutrophils having ingested other neutrophils during the course of coculture.

\section{Cytokine Analyses}

Sera from healthy controls, skin chamber fluids, synovial fluids and cell-free culture supernatants were analyzed for IL- $1 \beta$ content using a Bio-Plex Pro Cytokine assay (60 $\mu$ l, diluted 1:3; Bio-Rad Laboratories AB, Sundbyberg, Sweden) or a human IL-1 $\beta$ ELISA MAX Deluxe Set (BioLegend Inc., San Diego, Calif., USA), accord- ing to the manufacturer's descriptions. IL-1RA was measured using a human IL-1RA Quantikine ELISA (R\&D Systems), according to the manufacturer's description.

\section{Results}

\section{Neutrophils from Skin Chambers, but Not from \\ Synovial Fluid, Are Resistant to Antiapoptotic Stimulation}

We recently showed that in vivo transmigrated neutrophils, isolated from skin chambers of healthy volunteers, are unable to slow down their apoptotic rate in response to a wide variety of agents with potent antiapoptotic effects on neutrophils isolated from peripheral blood of the same subjects [5]. Here, we first confirmed these findings and showed that whereas apoptosis of neutrophils isolated from peripheral blood was effectively decreased by LPS ( $p=0.0008$, as compared to nonstimulated neutrophils using paired Student's $t$ test), apoptosis in neutrophils from skin chambers was unaffected by this treatment (fig. 1a). Also, spontaneous apoptosis was lower for skin chamber neutrophils as compared to blood neutrophils from the same donor $(\mathrm{p}=0.055)$ in this set of experiments (fig. 1a).

We next investigated whether resistance to antiapoptotic stimulation was a general feature of in vivo transmi- 


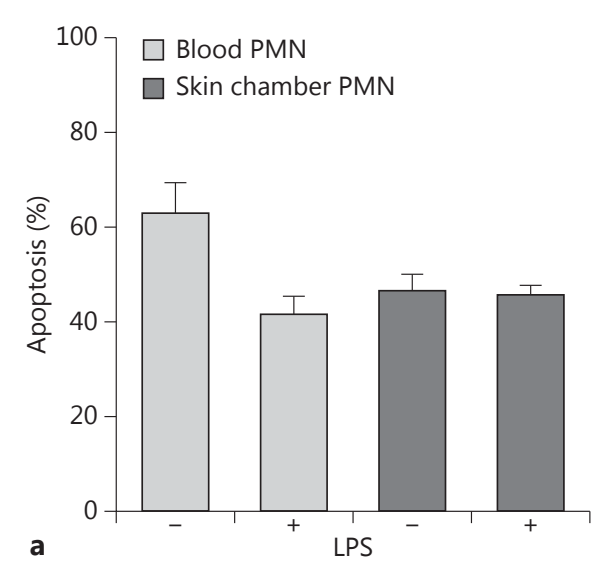

Fig. 1. Regulation of cell death in neutrophils from blood and tissue. PMN = Polymorphonuclear neutrophils. a Neutrophils from blood and skin chambers from the same subjects were cultured for $20 \mathrm{~h}$ in the presence or absence of LPS $(100 \mathrm{ng} / \mathrm{ml})$ after which apoptosis was quantified by annexin $\mathrm{V}$ staining. Bars show the mean + SEM from 6 healthy donors. $\mathbf{b}, \mathbf{c}$ Neutrophils from peripheral blood (b) and synovial fluid (c) of the same patients were cultured in vitro in the presence of a variety of antiapoptotic stimuli (LPS, $100 \mathrm{ng} / \mathrm{ml}$; rSAA, $2 \mu \mathrm{M}$; GM-CSF, 100 ng/ml; PGN, $10 \mu \mathrm{g} /$ $\mathrm{ml}$; LTA, $\left.10 \mu \mathrm{g} / \mathrm{ml} ; \mathrm{P}_{3} \mathrm{C}, 100 \mathrm{ng} / \mathrm{ml}\right)$, proapoptotic FASL $(10 \mu \mathrm{g} /$ $\mathrm{ml}$ ) or left unstimulated (us). After $20 \mathrm{~h}$ of incubation, neutrophil apoptosis was assessed by annexin $\mathrm{V}$ binding and flow cytometry. Graphs show means + SEM from 4-5 different patients.

grated neutrophils by studying cells obtained from synovial fluid (with a neutrophil content of $>65 \%$ ) of patients with inflammatory arthritis (table 1). We cultured neutrophils from blood and synovial fluid in vitro for $20 \mathrm{~h}$ with or without addition of established modulators of apoptosis. Surprisingly, spontaneous apoptosis was significantly elevated in synovial fluid neutrophils as compared to cells from peripheral blood of the same patients $(\mathrm{p}=0.0085$, using paired Student's t test), and both cell types increased in apoptosis in response to FASL (fig. 1b, c). Although levels of apoptosis were elevated overall in synovial fluid neutrophils, addition of antiapoptotic factors potently decreased cell death of these cells $(\mathrm{p}<0.05$ for all stimuli except $\mathrm{PGN}$ and $\mathrm{P}_{3} \mathrm{C}$, compared to unstimulated samples using one-way ANOVA followed by Dunnett's multiple comparison test) in a similar manner as
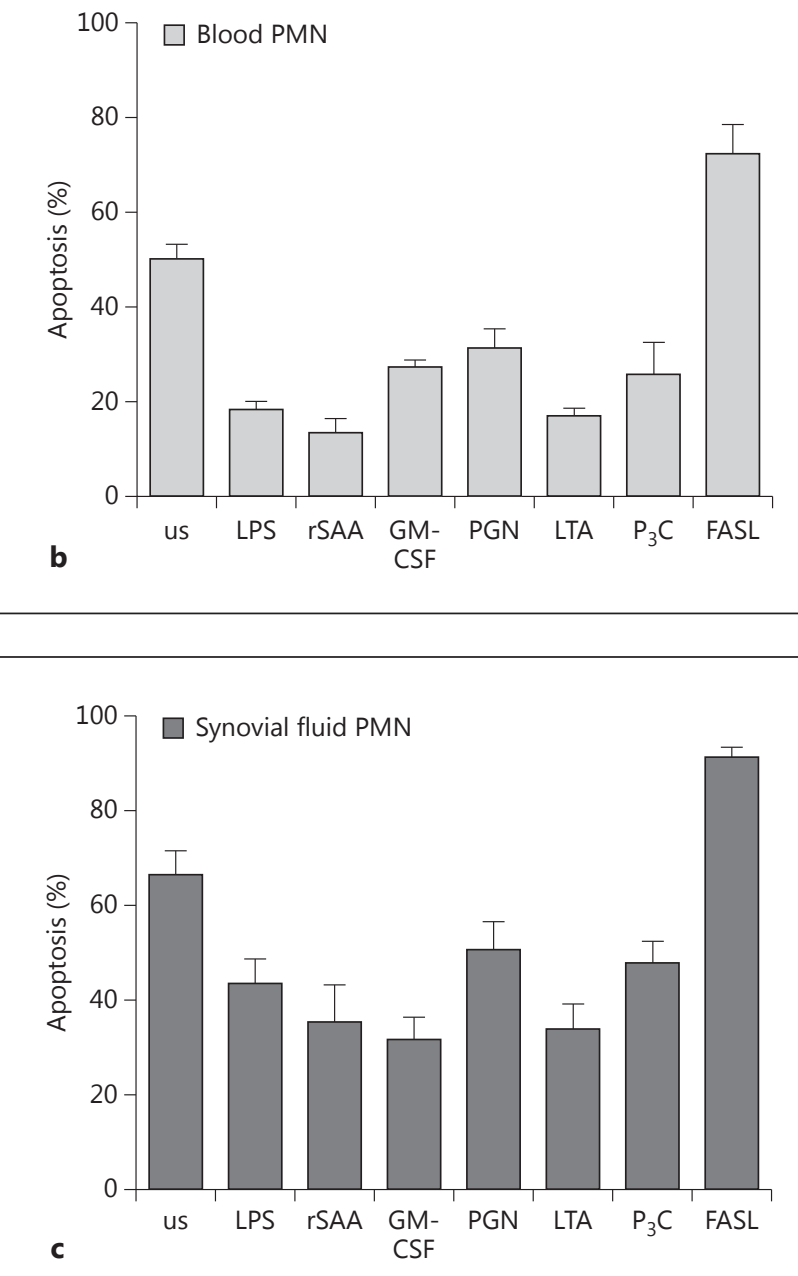

they delayed apoptosis of blood neutrophils ( $\mathrm{p}<0.05$ for all stimuli; fig. $1 \mathrm{~b}, \mathrm{c}$ ). Although the relative responses were somewhat less pronounced in synovial fluid neutrophils, these cells display a markedly different phenotype compared to skin chamber neutrophils. Thus, this demonstrates that the complete resistance to antiapoptotic stimulation exhibited by skin chamber neutrophils (fig. 1a) [5] is not a general feature of neutrophils that have left circulation.

\section{Freshly Prepared Synovial Fluid Neutrophils Display}

Signs of Ongoing Apoptosis

To better understand why neutrophils from synovial fluid demonstrated increased apoptosis as compared to blood neutrophils after $20 \mathrm{~h}$ of incubation, we analyzed these cells before culture (fig. 2). Microscopic examina- 
Fig. 2. Characterization of neutrophils from freshly aspirated synovial fluid. PMN = Polymorphonuclear neutrophils. a Scatter plots of freshly prepared blood neutrophils (left) and cells from freshly aspirated synovial fluid (right) from 1 representative patient out of 13. Cellular debris in the lower left corners (dotted squares) has been gated away, and the solid squares represent the neutrophil gates used for subsequent analyses. b Cell preparations were occasionally cytospun and stained with Giemsa/May-Grünwald solutions for microscopic evaluation. c Neutrophil viability of fresh preparations was monitored by annexin V/7-AAD staining and flow cytometry $(n=8)$. $\mathbf{d}$ The activity of caspase $3 / 7$ in the preparations was also determined immediately (fresh) and after $3.5 \mathrm{~h}$ of culture in the presence or absence of FASL $(10 \mu \mathrm{g} /$ $\mathrm{ml})$. Data are the mean + SEM $(\mathrm{n}=5)$. bPMN = Blood polymorphonuclear neutrophils; sfPMN = synovial fluid polymorphonuclear neutrophils; $\mathrm{AU}=$ arbitrary units.

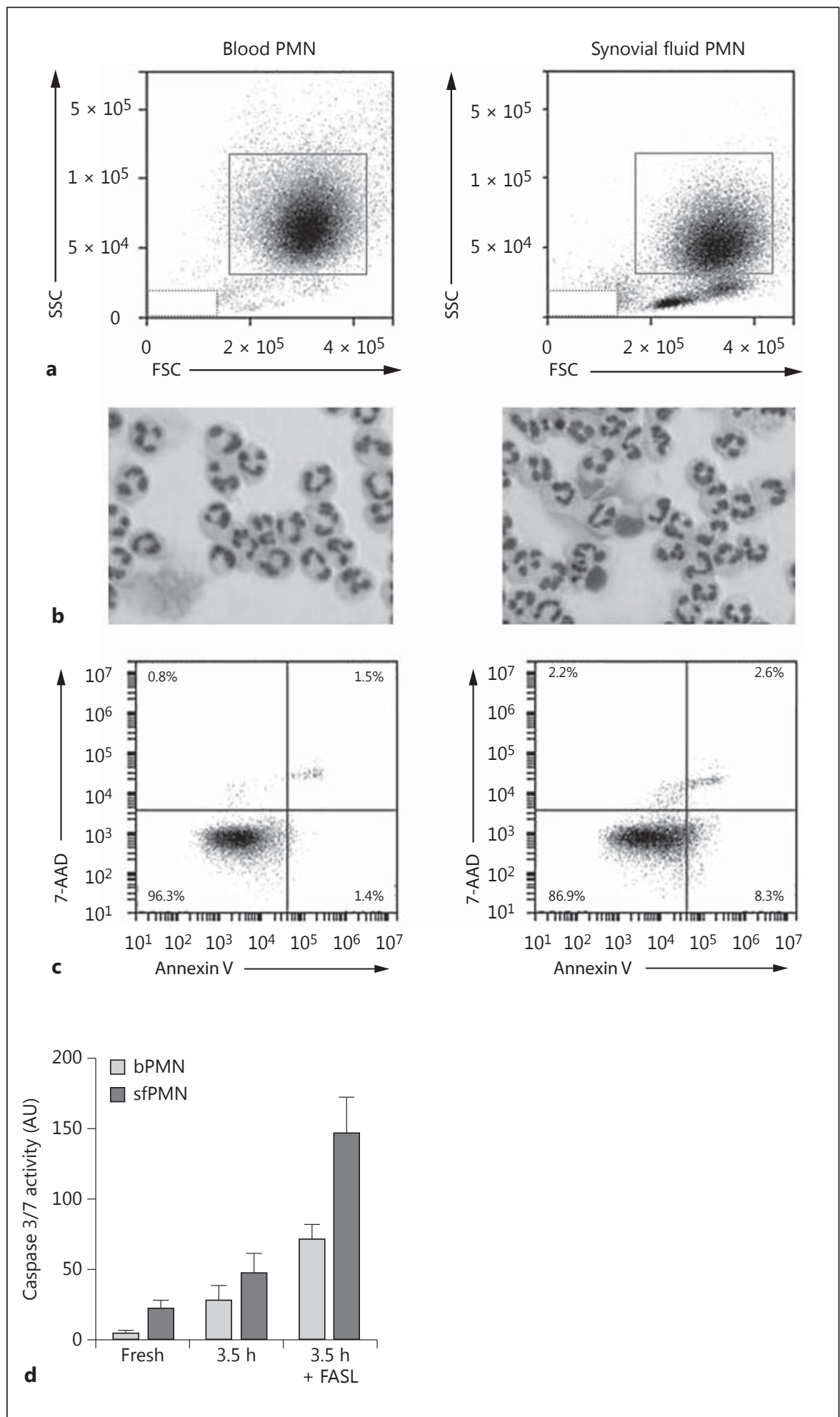




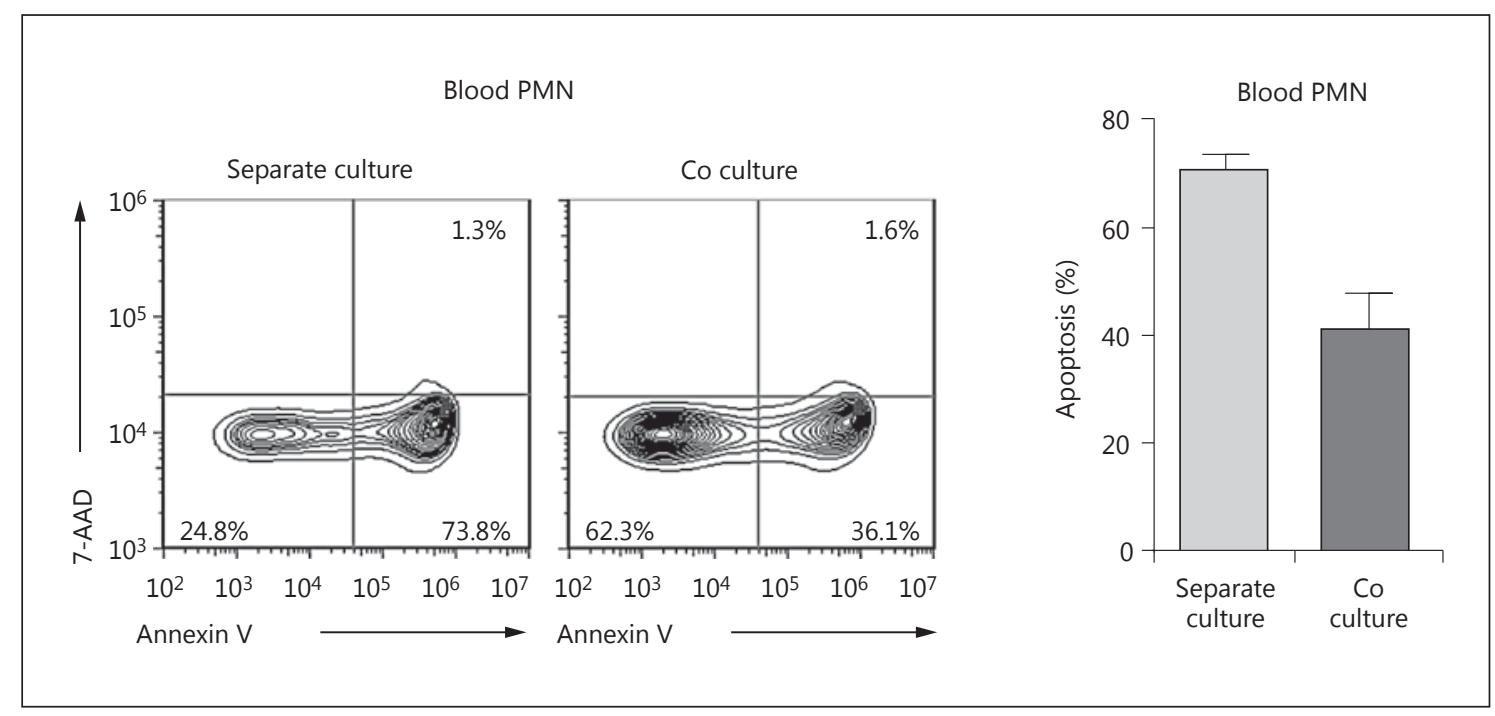

Fig. 3. Apoptosis of blood neutrophils in the presence or absence of skin chamber neutrophils. CFDA-labeled blood neutrophils were cultured alone or in the presence of unlabeled skin chamber neutrophils from the same donor. After $20 \mathrm{~h}$, apoptosis was assessed by annexin V staining after gating on the CFDA-positive blood neutrophils. One representative experiment is shown, as well as the mean + SEM of blood neutrophil apoptosis after separate cultures and cocultures, respectively $(n=5) . P M N=$ Polymorphonuclear neutrophils.

tion of freshly prepared synovial fluid cells showed mainly viable neutrophils with classic multilobulated nuclei (fig. 2b). Flow cytometry (fig. 2c) revealed slightly increased apoptosis in synovial fluid neutrophils as compared to blood neutrophils (annexin $\mathrm{V}$ positive/7-AAD negative: $6.7 \pm 4.2 \%$ in synovial fluid vs. $3.2 \pm 2.5 \%$ in neutrophils from blood; mean $\pm S D, n=8$ ). In line with this, we observed increased activity of caspase $3 / 7$, central for the apoptotic process, in synovial fluid neutrophils as compared to blood neutrophils, both when analyzed directly and after incubation in the presence or absence of FASL (fig. 2d). These data could likely explain why synovial fluid cells displayed increased overall apoptosis as compared to blood neutrophils.

\section{Skin Chamber Neutrophils Are Primed to Secrete Death-Delaying Signals}

As shown above (fig. 1c), synovial fluid neutrophils responded well to antiapoptotic stimulation, whereas the skin chamber neutrophils did not respond at all (fig. 1a) [5]. Since spontaneous apoptosis of the skin chamber neutrophils was lower as compared to blood neutrophils (fig. 1a), and the former are known to be primed for the production of proinflammatory cytokines [5, 14], we hypothesized that their slow apoptosis could be due to constant exposure to death-delaying agents acting in an au- tocrine fashion. To test this, we performed coculture experiments; after labeling peripheral blood neutrophil preparations with a cell tracker dye (CFDA), these cells were mixed with freshly prepared, unlabeled (and washed) skin chamber neutrophils from the same donor and cultured in vitro. The two cell types were easily distinguished, based on CFDA fluorescence, and the mere presence of skin chamber neutrophils significantly decreased apoptosis of blood neutrophils as compared to when these cells were cultured alone (fig. 3; $p=0.006$ using paired Student's t test). This indicates that the skin chamber cells are indeed primed to secrete antiapoptotic factor(s).

\section{Skin Chamber Neutrophils Secrete IL-1 $\beta$, a Cytokine with Antiapoptotic Properties}

As mentioned above, genes encoding a variety of proinflammatory cytokines are known to be upregulated in skin chamber neutrophils [4] as well as synthesized de novo $[5,6]$; some of these could potentially also be released without further stimulation. Skin chamber neutrophils spontaneously released markedly elevated levels of both IL-8 [5] (data not shown) and IL-1 $\beta$ (fig. 4a), as compared to blood neutrophils (fig. 4a). Recombinant IL-8 did not affect apoptosis, whereas IL- $1 \beta$ induced a dosedependent decrease in apoptosis of blood neutrophils in vitro (fig. $4 \mathrm{~b}, \mathrm{c}$ ). These results are in agreement with pre- 


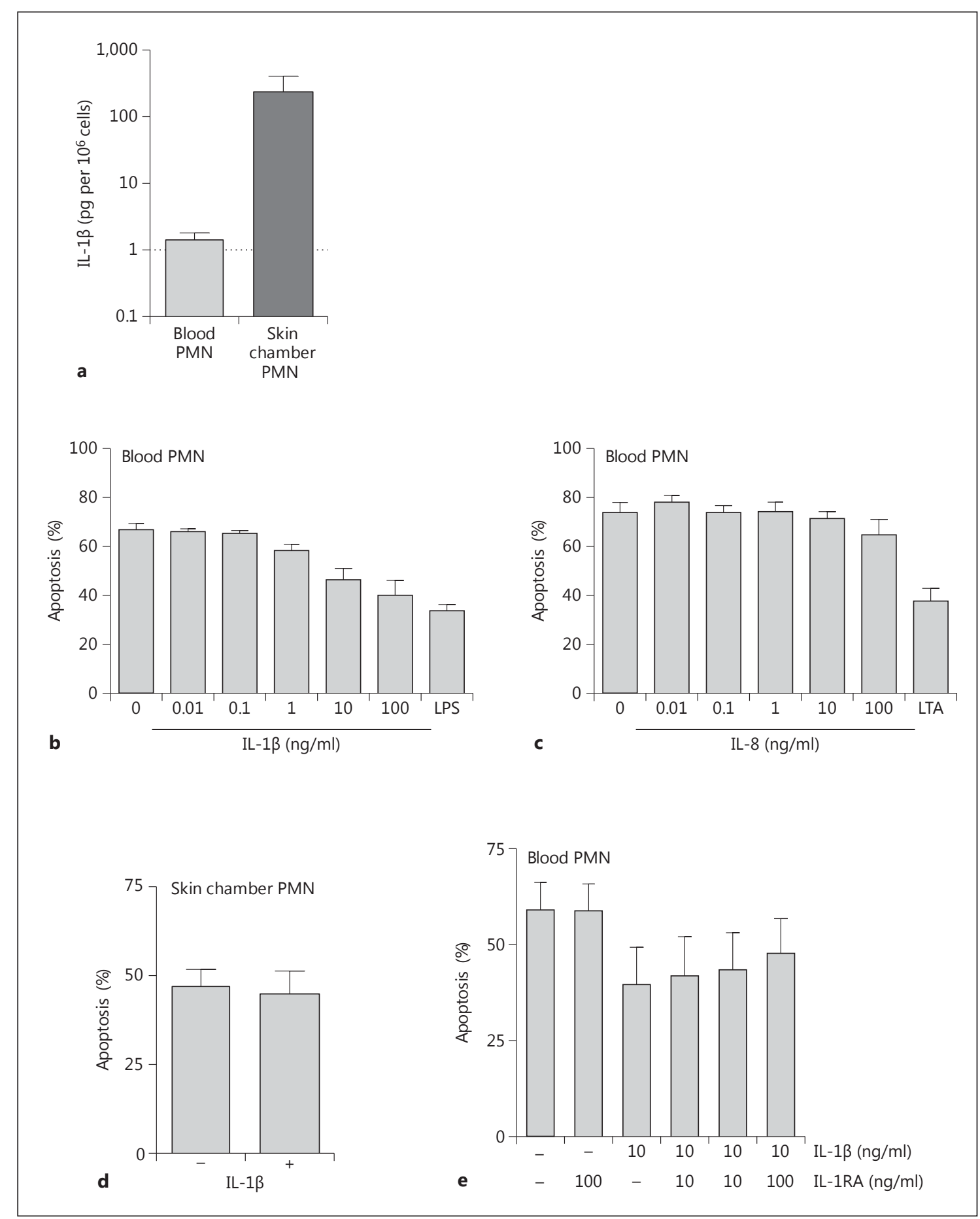

Fig. 4. Release of IL-1 $\beta$ and effects of this cytokine on apoptosis. PMN = Polymorphonuclear neutrophils. a Release of IL-1 $\beta$ from blood and skin chamber neutrophils was measured by ELISA after $24 \mathrm{~h}$ in vitro culture $(n=6)$. Bars are the mean + SEM, and the dotted line represents the lower detection limit of ELISA. b, c Neutrophils from peripheral blood were cultured in vitro with or without the addition of recombinant IL- $1 \beta$ (b) or IL- 8 (c) at indicated concentrations. LPS $(100 \mathrm{ng} / \mathrm{ml})$ and LTA $(10 \mu \mathrm{g} / \mathrm{ml})$ were used as positive controls, and data are shown as mean apoptosis + SEM $(\mathrm{n}=3)$. $\mathbf{d}$ Skin chamber neutrophils were cultured in the presence or absence of recombinant IL-1 $\beta(100 \mathrm{ng} / \mathrm{ml})$. Mean apoptosis + SEM $(n=5)$ is shown. e Blood neutrophils were incubated with or without a fixed concentration of recombinant IL-1 $\beta(10 \mathrm{ng} / \mathrm{ml})$ and indicated concentrations of IL-1RA. Data shown are mean apoptosis + SEM $(\mathrm{n}=4)$. 
Fig. 5. Soluble IL-1R ligands in serum, skin chamber fluid and synovial fluid. Levels of IL-1 $\beta$ (a) and IL-1RA (b) in control serum, skin chamber fluid and synovial fluid, as assessed by a multiplex assay or ELISA. Data are the mean + SEM from sera $(\mathbf{a}, \mathrm{n}=$ $4 ; \mathbf{b}, \mathrm{n}=5)$, skin chamber fluids $(\mathbf{a}, \mathrm{n}=4$; $\mathbf{b}, \mathrm{n}=3)$ and synovial fluids $(\mathbf{a}, \mathrm{n}=12 ; \mathbf{b}$, $\mathrm{n}=4$ ).

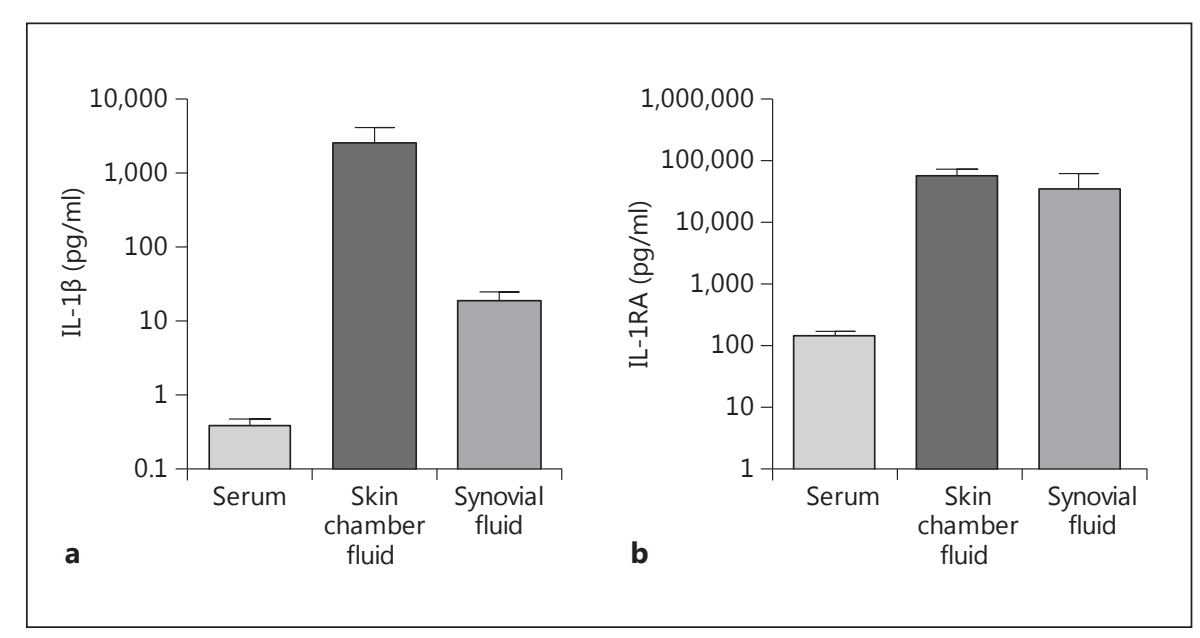

viously published data [15]. As could be expected from cells that continuously release IL- $1 \beta$, treatment with additional recombinant IL- $1 \beta$ to skin chamber neutrophils did not delay apoptosis (fig. 4d).

Of importance for the regulation of IL- $1 \beta$ signaling is IL-1RA, a competitive endogenous IL-1 receptor antagonist [16]. To test whether the antiapoptotic effects of IL$1 \beta$ were counteracted by IL-1RA, we added varying concentrations of recombinant IL-1RA to blood neutrophils stimulated with $10 \mathrm{ng} / \mathrm{ml}$ of IL- $1 \beta$. The results show a trend towards restoration of apoptosis with increasing concentrations of IL-1RA, but never to the level of the control, even at the highest possible IL-1RA concentration (fig. 4e). The IL-1RA did not lessen the antiapoptotic effect of LPS (data not shown), i.e. the action was specific for IL- $1 \beta$.

\section{Skin Chamber Fluid Is Characterized by a Higher}

\section{IL-1RA/IL-1 $\beta$ Ratio than Synovial Fluid}

Not only did skin chamber neutrophils in culture release IL-1 $\beta$ (fig. $4 \mathrm{a}$ ), we also found very high levels of this cytokine in the fluid from which these cells were obtained (fig. 5a). In fact, IL-1 $\beta$ levels in skin chamber fluid were much higher than those found in cell-free synovial fluid (fig. 5a). The levels of IL-1RA were similar in skin chamber fluid and synovial fluid, both elevated as compared to serum (fig. 5b). The IL-1RA/IL-1 $\beta$ ratio was thus markedly lower in skin chamber fluid as compared to synovial fluid, suggesting that skin chamber neutrophils, as opposed to synovial fluid neutrophils, come from a milieu of ongoing IL- $1 \beta$ signaling.

\section{Counteracting Inflammasome Activation and}

IL-1 $\beta$ Release from Skin Chamber Neutrophils Affects Apoptosis

Having concluded that skin chamber neutrophils spontaneously release antiapoptotic IL-1 $\beta$, we attempted to interfere with IL- $1 \beta$ signaling by using recombinant IL-1RA. In the coculture set-up, addition of IL-1RA slightly increased apoptosis of blood neutrophils, although it did not fully restore apoptosis back to the levels displayed by blood neutrophils cultured alone (fig. 6a).

The IL- $1 \beta$ release from neutrophils is known to depend on caspase 1 activity in the context of the NLRP3 inflammasome [17] and we next employed the caspase 1 inhibitor YVAD in order to diminish IL- $1 \beta$ secretion. Addition of YVAD decreased IL- $1 \beta$ release from skin chamber neutrophils, although not down to the undetectable levels secreted by blood neutrophils (fig. 6b). In line with this, YVAD also increased apoptosis of cocultured blood neutrophils in a separate set of experiments (fig. 6c). Neither IL-1RA nor YVAD had any effect on the apoptosis of blood neutrophils when cultured alone, indicating that caspase 1 and/or IL- $1 \beta$ is not involved in controlling spontaneous apoptosis of resting blood neutrophils.

Our data so far indicate that constitutive inflammasome-dependent IL- $1 \beta$ release from skin chamber neutrophils could function as a death-delaying signal for neighboring blood neutrophils during coculture. Thus, skin chamber neutrophils could be constantly affected by the antiapoptotic actions of endogenous IL- $1 \beta$, continuously released from these cells. If so, the spontaneous level of apoptosis of skin chamber neutrophils would in fact be underestimated. In order to test this, we decreased IL- 
Fig. 6. Effects of IL-1 $\beta$ antagonism or caspase 1 blockade on apoptosis. PMN = Polymorphonuclear neutrophils. a CFDA-labeled blood neutrophils were cultured alone or in coculture with skin chamber neutrophils from the same donor, in the presence or absence of recombinant IL1RA (100 ng/ml), after which apoptosis of blood neutrophils was measured. Graph shows mean apoptosis + SEM $(n=4)$. b Neutrophils from blood or skin chambers were cultured separately in the presence or absence of the caspase 1 inhibitor YVAD $(50 \mu \mathrm{M})$ after which the cell-free supernatants were analyzed for IL- $1 \beta$ content by ELISA. Values from blood neutrophil cultures were below detection limit (n.d.), and data are mean IL- $1 \beta+\operatorname{SEM}(n=3)$. c The experiment is a similar coculture setup as in a, but the neutrophils were incubated in the presence or absence of YVAD $(50 \mu \mathrm{M})$ before apoptosis was quantified. Mean apoptosis of blood neutrophils + SEM is shown $(\mathrm{n}=4)$. d Skin chamber neutrophils were cultured alone in the presence or absence of YVAD $(50 \mu \mathrm{M})$ after which apoptosis was quantified. The graph displays mean apoptosis + SEM $(n=4)$.

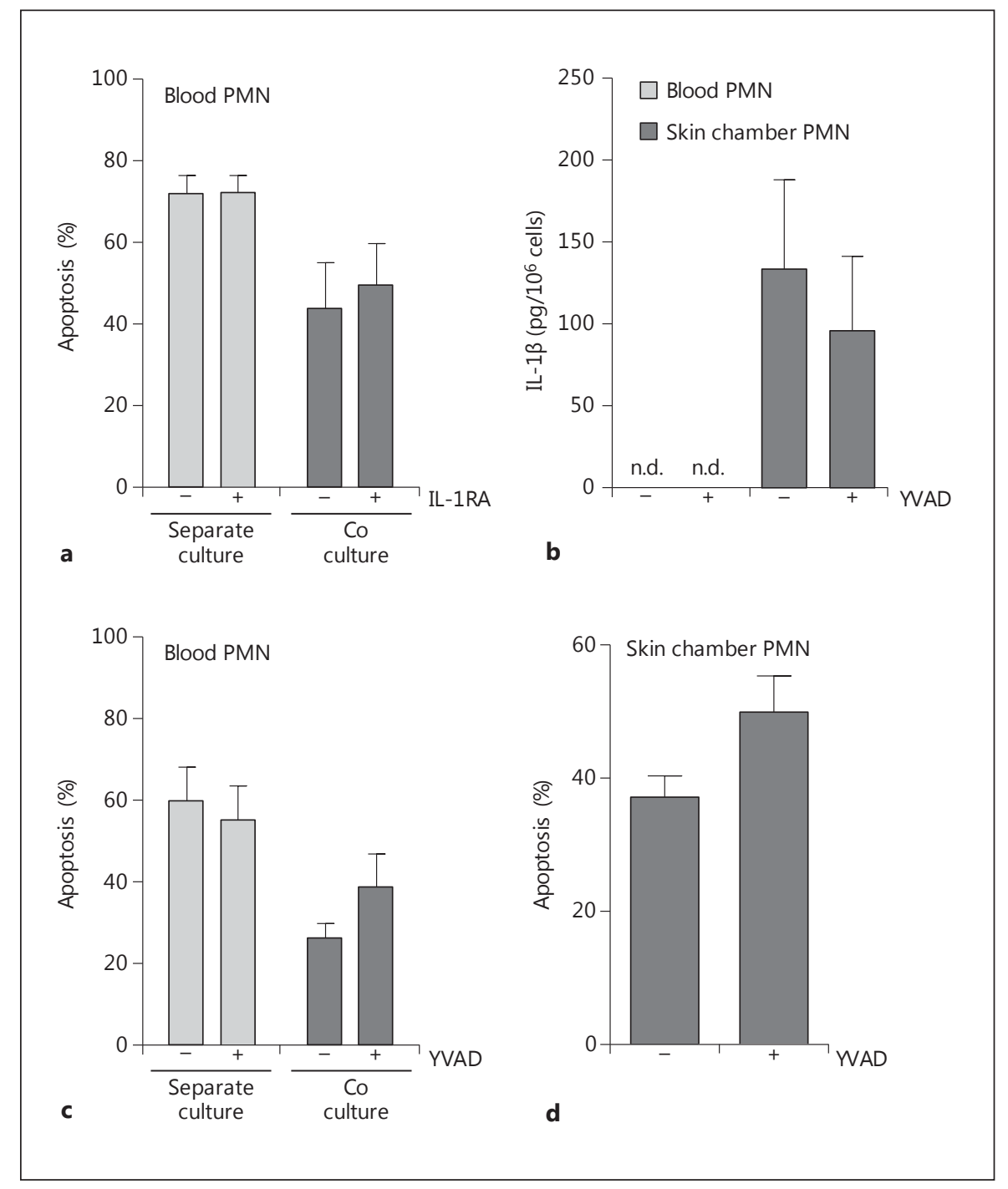

$1 \beta$ in the system by using YVAD. Blocking caspase 1 activity markedly increased spontaneous apoptosis of skin chamber neutrophils (fig. 6d), confirming that these cells are in fact under constant death suppression mediated by inflammasome-dependent IL- $1 \beta$ release.

\section{Discussion}

In vitro studies show that neutrophil cell death can be accelerated or postponed depending on environmental factors [10]. However, to what extent this modulation of apoptosis occurs in vivo is more difficult to evaluate. Neutrophils perform their major duties in extravascular tissues and it has previously been described that the trans- migration process from blood to tissue renders neutrophils in a preactivated, or primed, state $[14,18]$. It is reasonable to believe that cellular priming also affects neutrophil apoptosis. Therefore, investigations of possible differences in cell death between neutrophils obtained from peripheral blood and after transmigration to tissue are of interest. The current study corroborated our previous finding [5] that skin chamber neutrophils are unable to extend their viability in response to antiapoptotic factors and further demonstrate that skin chamber neutrophils constantly produce and secrete factors that can delay apoptosis of blood neutrophils. We found that IL- $1 \beta$ was secreted by skin chamber neutrophils and displayed antiapoptotic properties when added to blood neutrophils in vitro. 
We also investigated how in vivo transmigrated neutrophils from a pathologically inflamed site regulated apoptosis by utilizing synovial fluid aspirated from patients diagnosed with different forms of inflammatory arthritis (table 1). These disorders are characterized by a state of chronic inflammation, but during flares of active disease, an additional component of acute inflammation of the joints results in swelling and transmigration of leukocytes to this otherwise acellular site. The cellular composition of the inflamed synovial fluid varies, with neutrophils often being the dominating cell type; only patients with $>65 \%$ neutrophils in the synovial fluid were included in this study. One drawback of using synovial fluid instead of the relatively controlled skin chamber model is that it is impossible to determine the age of the cells isolated and to control for how long the transmigrated neutrophils have lingered in the joint. The synovial fluid neutrophils displayed elevated caspase $3 / 7$ activity and annexin $\mathrm{V}$ binding already when aspirated and were also more inclined to spontaneous apoptosis after in vitro culture. These data imply that the synovial fluid neutrophils are in fact aged as compared to the cells isolated from peripheral blood of the same patients, which could explain why apoptosis of the former was increased after in vitro culture.

Inflamed synovial fluid has been described to be a relatively rich source of inflammatory mediators [19], and several studies have evaluated the impact of cell-free synovial fluid on neutrophil survival. Most of these studies have been performed with blood neutrophils, and conclusions are in conflict $[20,21]$. Discrepancies could possibly stem from the fact that the inflamed synovia is hypoxic $[22,23]$ and that pro- or antiapoptotic factors in synovial fluid dominate under different oxygen tension [24]. Another aspect to consider is that the synovial fluids were aspirated from patients under treatment with a variety of anti-inflammatory drugs (table 1). Some of these drugs have been shown to elevate neutrophil apoptosis in vitro [25] and in vivo [26]. However, blood neutrophils from the patients included in our study responded similarly to modulators of apoptosis as did cells from healthy individuals, indicating that the anti-inflammatory therapy per se did not directly influence our results.

Neutrophils obtained from synovial fluid were fully able to postpone apoptosis in response to stimulation with a variety of antiapoptotic factors. This is in glaring contrast to the skin chamber neutrophils that clearly lack this ability (fig. 1) [5]. The differences in response to antiapoptotic stimulation between the two types of tissue neutrophils likely reflect the nature of the underlying in- flammation. Whereas skin chamber neutrophils migrate towards an aseptic, acute inflammation in an otherwise healthy tissue, transmigration to synovial fluid is a much more complex scenario. Affected joints in inflammatory arthritis represent tissues with a pathological, chronic inflammation, and added to this is an acute inflammatory response as seen by e.g. joint swelling, leukocyte infiltration and enhanced sedimentation rate. One major difference between neutrophils from skin chambers and synovial fluid was that they were taken from sites with markedly different IL- $1 \beta$ levels. Whereas levels of IL-1RA were relatively similar, skin chamber fluid contained almost 200 times more IL-1 $\beta$ than did synovial fluid. The skin chamber neutrophils spontaneously produced and released this particular cytokine also during in vitro culture. Although we cannot rule out that some of the IL- $1 \beta$ originate from other cells, human neutrophils have been shown to produce substantial amounts of IL- $1 \beta$ [17], and upon transmigration to skin, both the gene [4] and protein [27] are significantly upregulated specifically in these cells. The IL-1 $\beta$ release was partly suppressed by inhibition of caspase 1 , which implies that transmigration to skin chambers induces continuous inflammasome activation.

The NALP3 inflammasome has been the focus of much attention in recent years and the name refers to a protein complex that relies on caspase 1 to process IL- $1 \beta$ and IL-18 from proforms into active cytokines that are released extracellularly [28]. Certain kinds of nonapoptotic cell death modes (e.g., pyroptosis and pyronecrosis) rely on NALP3 inflammasome activity [29], and these are characterized not only by IL- $1 \beta$ release, but also by loss of membrane integrity of the affected cells [29]. We never experienced more than a few percent of cells with permeable membranes in our study. In addition, blocking caspase 1 never decreased cell death, indicating that these types of nonapoptotic death processes were of little or no significance in our experimental set-ups. Contrarily, the caspase 1 inhibitor YVAD actually increased apoptosis in blood neutrophils when cultured in the presence of skin chamber neutrophils. This fits well with the idea that these in vivo transmigrated neutrophils spontaneously secrete IL-1 $\beta$ that suppresses apoptosis of neighboring blood cells when cultured together. The fact that YVAD only partially blocked IL- $1 \beta$ secretion from skin chamber neutrophils is in line with the existence of caspase-1-independent mechanisms for IL- $1 \beta$ processing [30], which could explain why apoptosis of cocultured blood neutrophils was not completely restored. 
The other inflammasome-dependent cytokine secreted by neutrophils is IL-18, and although we never studied IL-18 further, previous reports argue that it is without effects on neutrophil apoptosis [31]. The use of recombinant IL-1RA never fully neutralized the antiapoptotic effect of recombinant IL- $1 \beta$, and this reagent also failed to fully restore apoptosis of cocultured blood neutrophils. However, together with the effects of the caspase 1 inhibitor, our data indicate that inflammasome-mediated IL- $1 \beta$ release from skin chamber neutrophils constitutes a death-delaying signal even though we cannot completely rule out that also other signaling routes and soluble mediators play a role. One candidate in this respect would be IL- 8 which is avidly produced and secreted by skin chamber neutrophils $[7,32,33]$. However, this particular cytokine was not antiapoptotic in our hands despite possessing full biological activity in other assays (e.g., the induction of neutrophil degranulation, calcium signaling and chemotaxis at $1-100 \mathrm{ng} / \mathrm{ml}$ concentrations; data not shown). Although this may be a matter of some controversy, the inability of recombinant IL-8 to delay neutrophil apoptosis confirms earlier reports [15].

Recombinant IL-1 $\beta$ did not alter apoptosis of skin chamber neutrophils, but limiting the release of endogenous IL- $1 \beta$ by YVAD, resulted in increased apoptosis of these cells. This suggests that skin chamber neutrophils are constantly subjected to antiapoptotic stimulation by endogenous IL- $1 \beta$ released in an inflammasome-dependent manner. In line with this, a phenotype reminiscent of the skin chamber neutrophils was recently described for (blood-derived) neutrophils from patients carrying two inflammasome-associated gain-of-function mutations. These cells display constitutive inflammasome activation and IL- $1 \beta$ secretion, but also exhibit a suppressed apoptotic rate [34]. Autocrine IL-1 $\beta$ signaling would make additional exogenous IL- $1 \beta$ useless and it may well be that the 'spontaneous apoptosis' (i.e., in the absence of exogenous stimulation) displayed by skin chamber neutrophils is in reality a process already slowed down by endogenous IL-1 $\beta$. Such autocrine death suppression could potentially mask responses to death-delaying substances added to in vitro cultures. In summary, we describe here that neutrophil apoptosis is regulated differently depending on the site and inflammatory setting into which the cells transmigrate. Our data further imply that inflammasome-mediated secretion of IL- $1 \beta$ could be an important factor in determining the life span of neutrophils at certain inflamed sites. In light of these data, future studies on synovial fluid neutrophils obtained from rheumatic patients where inflammasome activation has been specifically documented, such as gout [35], would be of great interest.

\section{Acknowledgements}

This work was supported by the Swedish Research Council (521-2009-3443), the King Gustav V Memorial Foundation, Gothenburg Medical Society, Ingabritt and Arne Lundgren's Research Foundation, the Gothenburg Rheumatism Association, and the Swedish State under the LUA/ALF agreement.

\section{References}

1 Zarember KA, Kuhns DB: Editorial: will the real neutrophil please stand up? J Leuk Biol 2011;90:1039-1041.

-2 Bylund J, Karlsson A, Boulay F, Dahlgren C: Lipopolysaccharide-induced granule mobilization and priming of the neutrophil response to Helicobacter pylori peptide $\mathrm{HP}(2-20)$, which activates formyl peptide receptor-like 1. Infect Immun 2002;70:2908-2914.

$\checkmark 3$ Karlsson A, Follin P, Leffler H, Dahlgren C: Galectin-3 activates the NADPH-oxidase in exudated but not peripheral blood neutrophils. Blood 1998;91:3430-3438.

4 Theilgaard-Monch K, Knudsen S, Follin P, Borregaard N: The transcriptional activation program of human neutrophils in skin lesions supports their important role in wound healing. J Immunol 2004;172:7684-7693.
5 Christenson K, Bjorkman L, Karlsson J, Sundqvist M, Movitz C, Speert DP, Dahlgren C, Bylund J: In vivo-transmigrated human neutrophils are resistant to antiapoptotic stimulation. J Leukoc Biol 2011;90:1055-1063.

$\checkmark 6$ Follin P: Skin chamber technique for study of in vivo exudated human neutrophils. J Immunol Methods 1999;232:55-65.

7 Kuhns DB, DeCarlo E, Hawk DM, Gallin JI: Dynamics of the cellular and humoral components of the inflammatory response elicited in skin blisters in humans. J Clin Invest 1992; 89:1734-1740.

8 Gregory CD, Devitt A: The macrophage and the apoptotic cell: an innate immune interaction viewed simplistically? Immunology 2004;113:1-14.

$>9$ Savill J, Dransfield I, Gregory C, Haslett C: A blast from the past: clearance of apoptotic cells regulates immune responses. Nat Rev Immunol 2002;2:965-975.
10 Simon HU: Neutrophil apoptosis pathways and their modifications in inflammation. Immunol Rev 2003;193:101-110.

11 Christenson K, Thoren FB, Bylund J: Analyzing cell death events in cultured leukocytes. Methods Mol Biol 2012;844:65-86.

12 Böyum A: Isolation of mononuclear cells and granulocytes from human blood. Isolation of monuclear cells by one centrifugation, and of granulocytes by combining centrifugation and sedimentation at $1 \mathrm{~g}$. Scand J Clin Lab Invest Suppl 1968;97:77-89.

$>13$ Boyum A, Lovhaug D, Tresland L, Nordlie EM: Separation of leucocytes: improved cell purity by fine adjustments of gradient medium density and osmolality. Scand J Immunol 1991;34:697-712. 
14 Sengelov H, Follin P, Kjeldsen L, Lollike K, Dahlgren C, Borregaard N: Mobilization of granules and secretory vesicles during in vivo exudation of human neutrophils. J Immunol 1995;154:4157-4165.

15 Colotta F, Re F, Polentarutti N, Sozzani S, Mantovani A: Modulation of granulocyte survival and programmed cell death by cytokines and bacterial products. Blood 1992;80:20122020.

-16 Bellehumeur C, Blanchet J, Fontaine JY, Bourcier N, Akoum A: Interleukin 1 regulates its own receptors in human endometrial cells via distinct mechanisms. Hum Reprod 2009, 24:2193-2204.

$\checkmark 17$ Mankan AK, Dau T, Jenne D, Hornung V: The NLRP3/ASC/Caspase- 1 axis regulates IL1 beta processing in neutrophils. Eur J Immunol 2012;42:710-715.

18 Paulsson JM, Jacobson SH, Lundahl J: Neutrophil activation during transmigration in vivo and in vitro. A translational study using the skin chamber model. J Immunol Methods 2010;361:82-88.

19 Koch AE, Kunkel SL, Shah MR, Hosaka S, Halloran MM, Haines GK, Burdick MD, Pope RM, Strieter RM: Growth-related gene product alpha. A chemotactic cytokine for neutrophils in rheumatoid arthritis. J Immunol 1995; 155:3660-3666.

20 Bell AL, Magill MK, McKane R, Irvine AE: Human blood and synovial fluid neutrophils cultured in vitro undergo programmed cell death which is promoted by the addition of synovial fluid. Ann Rheum Dis 1995;54:910915.
1 Ottonello L, Cutolo M, Frumento G, Arduino N, Bertolotto M, Mancini M, Sottofattori E, Dallegri F: Synovial fluid from patients with rheumatoid arthritis inhibits neutrophil apoptosis: role of adenosine and proinflammatory cytokines. Rheumatology (Oxford) 2002;41:1249-1260.

22 Bodamyali T, Stevens CR, Billingham ME, Ohta S, Blake DR: Influence of hypoxia in inflammatory synovitis. Ann Rheum Dis 1998; 57:703-710.

23 Mapp PI, Grootveld MC, Blake DR: Hypoxia, oxidative stress and rheumatoid arthritis. $\mathrm{Br}$ Med Bull 1995;51:419-436.

24 Cross A, Barnes T, Bucknall RC, Edwards SW, Moots RJ: Neutrophil apoptosis in rheumatoid arthritis is regulated by local oxygen tensions within joints. J Leukoc Biol 2006;80: 521-528.

25 Derouet M, Thomas L, Moulding DA, Akgul C, Cross A, Moots RJ, Edwards SW: Sodium salicylate promotes neutrophil apoptosis by stimulating caspase-dependent turnover of Mcl-1. J Immunol 2006;176:957-965.

26 Weinmann P, Moura RA, Caetano-Lopes JR, Pereira PA, Canhao H, Queiroz MV, Fonseca JE: Delayed neutrophil apoptosis in very early rheumatoid arthritis patients is abrogated by methotrexate therapy. Clin Exp Rheumatol 2007;25:885-887.

Basran A, Jabeen M, Bingle L, Stokes CA, Dockrell DH, Whyte MK, Walmsley SR, Higgins KR, Vogel SN, Wilson HL, Prince LR, Prestwich EC, Sabroe RA, Parker LC, Sabroe I: Roles of neutrophils in the regulation of the extent of human inflammation through delivery of IL-1 and clearance of chemokines. J Leukoc Biol 2013;93:7-19.
28 Martinon F, Mayor A, Tschopp J: The inflammasomes: guardians of the body. Annu Rev Immunol 2009;27:229-265.

29 Ting JP, Willingham SB, Bergstralh DT: NLRs at the intersection of cell death and immunity. Nat Rev Immunol 2008;8:372-379.

- 30 Netea MG, Simon A, van de Veerdonk F, Kullberg BJ, Van der Meer JW, Joosten LA: IL-1beta processing in host defense: beyond the inflammasomes. PLoS Pathog 2010; 6:e1000661

-31 Costantini C, Micheletti A, Calzetti F, Perbellini O, Pizzolo G, Cassatella MA: Neutrophil activation and survival are modulated by interaction with NK cells. Int Immunol 2010;22: 827-838.

32 Follin P, Wymann MP, Dewald B, Ceska M, Dahlgren C: Human neutrophil migration into skin chambers is associated with production of NAP-1/IL8 and C5a. Eur J Haematol 1991;47:71-76.

33 Lundahl J, Jacobson SH, Paulsson JM: IL-8 from local subcutaneous wounds regulates CD11B activation. Scand J Immunol 2012;75: 419-425.

34 Blomgran R, Patcha Brodin V, Verma D, Bergstrom I, Soderkvist P, Sjowall C, Eriksson P, Lerm M, Stendahl O, Sarndahl E: Common genetic variations in the NALP3 inflammasome are associated with delayed apoptosis of human neutrophils. PloS One 2012;7:e31326.

- 35 Martinon F, Petrilli V, Mayor A, Tardivel A, Tschopp J: Gout-associated uric acid crystals activate the NALP3 inflammasome. Nature 2006;440:237-241. 